\title{
The Potential Effect of Coconut Water as Natural Healer in Adult Patient with Chronic Periodontitis: A Preliminary Clinical Study
}

Zurainie Abllah¹, Syarifah Nurul Afifah Syed Ibrahim², Hamiza A Rahaman², Munirah Yaacob ${ }^{3}$, Yunita Dewi Ardini ${ }^{1}$

${ }^{1}$ Department of Paediatric Dentistry and Dental Public Health, Kulliyyah of Dentistry, International Islamic University Malaysia, Kuantan, Pahang

${ }^{2}$ Kulliyyah of Dentistry, International Islamic University Malaysia, Kuantan Pahang ${ }^{3}$ Department of Restorative Dentistry, Kulliyyah of Dentistry, International Islamic University Malaysia, Kuantan Pahang

Introduction: Mechanical and chemical debridement had been practiced as one of the management for chronic periodontitis. Recent interest and advances in the field of alternative medicine has promoted the use of various herbal and natural products for multiple uses in the field of medicine. As for it, coconut water is one of the potential natural sources that have various health benefits. Materials and method: Randomized control study was chosen as study design in this research project. 12 patients who have moderate to severe chronic periodontitis were selected from patients whom attended Polyclinic Kulliyyah of Dentistry, IIUM Kuantan Campus from November 2016 to January 2018. After stratified random sampling done, the subjects divided into 2 groups; Intervention Group and Control Group. All patients will go for ultrasonic scaling and root planning by using hand instruments. 6 patients in Intervention Group were given fresh coconut water to drink during daytime. The first re-assessment was done after 6 weeks. The control group of another 6 patients didn't receive any medicament. Paired sample t-test was used to analyse the pre and post intervention data. The study had obtained ethical approval from IREC (IREC 849). Results: Probing depth (PD) at mid-buccal site and bleeding on probing (BoP) shown significant result $(\mathrm{p}<0.05)$ in intervention group. There is no significant difference noted in the control groups, which are for PD, BoP and Plaque Score (PS) variables. Conclusion: Coconut water has potential benefit in reducing the periodontal pockets with chronic periodontitis as an adjunct after mechanical debridement. 